\title{
Quality Control of Selected Antimalarials Sold in the Illicit Market: An Investigation Conducted in Porto-Novo City (Republic of Benin)
}

\author{
Farid Baba-Moussa1,2, Jacob Bonou ${ }^{1,2}$, Hélène Ahouandjinou1,2, \\ Tamègnon Victorien Dougnon ${ }^{3}$, Lisette Kpavode ${ }^{1}$, Nawal Raimi ${ }^{1}$, \\ Aurore Ogouyemi-Hounto ${ }^{4}$, Idrissou Abdoulaye ${ }^{5}$, Fatiou Toukourou2 ${ }^{2}$, Frédéric Loko3, \\ Dorothée Kindé-Gazard $^{4}$, Flore Gangbo ${ }^{6}$, Lamine Baba-Moussa ${ }^{7 *}$ \\ ${ }^{1}$ National laboratory Control of Quality of Medicines and Edible Medical, Ministry of Health, Cotonou, Benin \\ ${ }^{2}$ Laboratory of Microbiology and Food Technology, Faculty of Sciences and Techniques, University of \\ Abomey-Calavi, Institute of Applied Biomedical Sciences, Champ de Foire, Cotonou, Benin \\ ${ }^{3}$ Research Laboratory in Applied Biology, Polytechnic School of Abomey-Calavi, University of Abomey-Calavi, \\ Cotonou, Benin \\ ${ }^{4}$ Parasitology and Mycology Laboratory, Faculty of Health Sciences, University of Abomey-Calavi, Cotonou, \\ Benin \\ ${ }^{5}$ Biochemistry Laboratory, Faculty of Health Sciences, CNHU-HKM, Cotonou, Benin \\ ${ }^{6}$ Cytogenetics and Human Biology Laboratory, ISBA-Champ de Foire, Cotonou, Benin \\ ${ }^{7}$ Laboratory of Biology and Molecular Typing in Microbiology, Faculty of Sciences and Techniques, University of \\ Abomey-Calavi, Cotonou, Benin \\ Email: "laminesaid@yahoo.fr
}

Received 31 August 2015; accepted 24 October 2015; published 27 October 2015

Copyright (C) 2015 by authors and Scientific Research Publishing Inc.

This work is licensed under the Creative Commons Attribution International License (CC BY).

http://creativecommons.org/licenses/by/4.0/

c) (i) Open Access

\section{Abstract}

Malaria is a parasitic disease caused by the bite of female Anopheles mosquito and particularly affects the tropical areas of the world. According to national statistics it is the leading cause of consultations and hospitalizations. Nowadays, despite the surveillance systems for efficient malaria control and access to generic drugs, Benin is witnessing an increased development of illicit drug markets with a large part of the population going towards such markets. However, this is not without adverse impact on the health of individuals, as well as, the economic status of the country. Therefore, the situation needs to be seriously considered by policy makers at various levels, health professionals but also the entire international community in order to thwart this scourge.

\footnotetext{
${ }^{*}$ Corresponding author.
} 
Regarding the aforementioned situation, the current study was undertaken aiming to perform a quality control of selected antimalarial drugs of the illegal market in Porto-Novo city. Therefore, 40 antimalarial batches were randomly collected in the illicit drug market and submitted to analytical tests such as: macroscopic examination (a visual and critical examination); mass uniformity test; disintegration test; identification test and active ingredients' content measurement. At the end of the study, the percentages of non-compliance is $97.5 \%, 5 \%, 15 \%$ and $27.5 \%$, respectively for the content uniformity tests, disintegration, identification and assay. Over-all, $42.5 \%$ of noncompliance was recorded. The findings of this study prove that street vended drugs offer no guarantee of good quality and pose a threat to the health of populations. Also, the rate of non-compliance denotes a flaw in the security of the drug distribution system.

\section{Keywords}

\section{Antimalarial, Counterfeit Medicine, Quality Control, Illicit Market}

\section{Introduction}

Malaria particularity affects poor tropical areas of Africa, Asia and Latin America representing in total 2.4 billion people, or $40 \%$ of the world population. Currently, 300 to 500 million patients are affected with 1.5 to 2.7 million deaths per year. Malaria remains the most important tropical parasitic disease. Over $90 \%$ of malaria cases and deaths are concentrated in sub-Saharan Africa [1]. The disease has taken a heavy toll among children under 5, pregnant women and new born ones [2]. According to WHO, in Africa malaria kills a child every 30 seconds, and represents $10 \%$ of the total disease burden on the continent, $40 \%$ of public health expenditures and more than $\$ 12$ billion loss in the Gross Domestic Product (GDP) [3].

In Benin, malaria remains a major public health problem and hinders the development of the country through the loss of lives and work days [4]. National statistics show that malaria is the leading cause of consultations and hospitalizations with a rate of $39.6 \%$, the first cause of consultation in children under 5 with a rate of $43.5 \%$ and the main cause of death in health facilities with a rate of $22.2 \%$ among children under five [5].

The World Health Organization [6] estimated that each year, two hundred thousand $(200,000)$ patients with malaria die because of poor quality drugs [7]. Indeed, the effectiveness of an antimalarial depends not only on the specific action that it exercises but also its quality and safety.

In fact, drug is not a product like any other because its importation, prescription and dispensation are strictly reserved for medical professionals [8]. Also, the commercialization of drug requires prior Marketing Authorisation issued by the Ministry of Health.

Therefore, a part from the qualified and authorized structures and health professionals, antimalarials are not supposed to be imported, prescribed or dispensed by street vendors. Besides, as this latter is unaware of any notion about the drugs including its mechanism of action, its dosage and its adverse effects, he or she should not play a role of dispenser or so. Unfortunately, children under five, pregnant women and elders are the most exposed people of the population to the consequences of the acts of these informal drug suppliers. The ineffectiveness of such treatment will not only influence the health status of the patient but also impact negatively the family budget, since unmet treatment will push consumers to procure more. As in other developing countries, Benin is characterized by porous borders, corruption, lack of technical and financial means for the detection of counterfeit drugs, lack of vigilance of the pharmaceutical system, tolerance of the informal sector etc. All these negative conditions can favour the emergence of illegal trade in drugs.

Antimalarials, essential drugs in developing countries become a danger to public health when they come from an informal system because they are not subjected to any quality control. These counterfeit antimalarial medicines are often over-dosed, under-dosed and some without active ingredient or may contain other toxic substances to consumer's body.

In Benin, a pilot study conducted in 2010 on the quality control of some antimalarial drugs sold in the unofficial market Adjégounlè (Dantokpa market), showcase of the informal sector, reported that only one third of artemether-lumefantrine samples were in accordance with the standards of the pharmacopoeia while none of sulfadoxine-pyrimethamine samples, was compliant [9]. 
With respect to such results, the current study was undertaken to extend the scope of the study on counterfeit antimalarials in Porto-Novo city. Indeed, Porto-Novo is a city which borders Nigeria, a country that is known for the importation of many low quality products especially drugs. Also, no published data exist about this issue in this region despite all the consequences aforementioned.

\section{Methodology}

This study was carried out from April to December 2011. Drug samples were collected by the research team who were introduced in the market as resellers so as to avoid suspicions from sellers and thus eliminate bias. As the size of illegally vended antimalarials was not known in advance, 10 batches of quinine, sulfadoxine-pyrimethamine, artemether-lumefantrine and artesunate-amodiaquine were collected. The reference substances for each sampled molecules were used as control. For sample analysis, the working protocol of the National Laboratory for Quality Control of Benin (LNCQ) supplemented by methods in different pharmacopoeias [10]-[13] was used. The analytical steps were performed according to the diagram in Figure 1.

\section{QUESTIONS}

External appearance and packaging complies?

The drug is able to deliver its content?

The drug contains the expected active(s) substance(s)?

The drug contains an amount of active ingredient conform to the expected dosage? The drug is free of detectable impurity?
TESTS

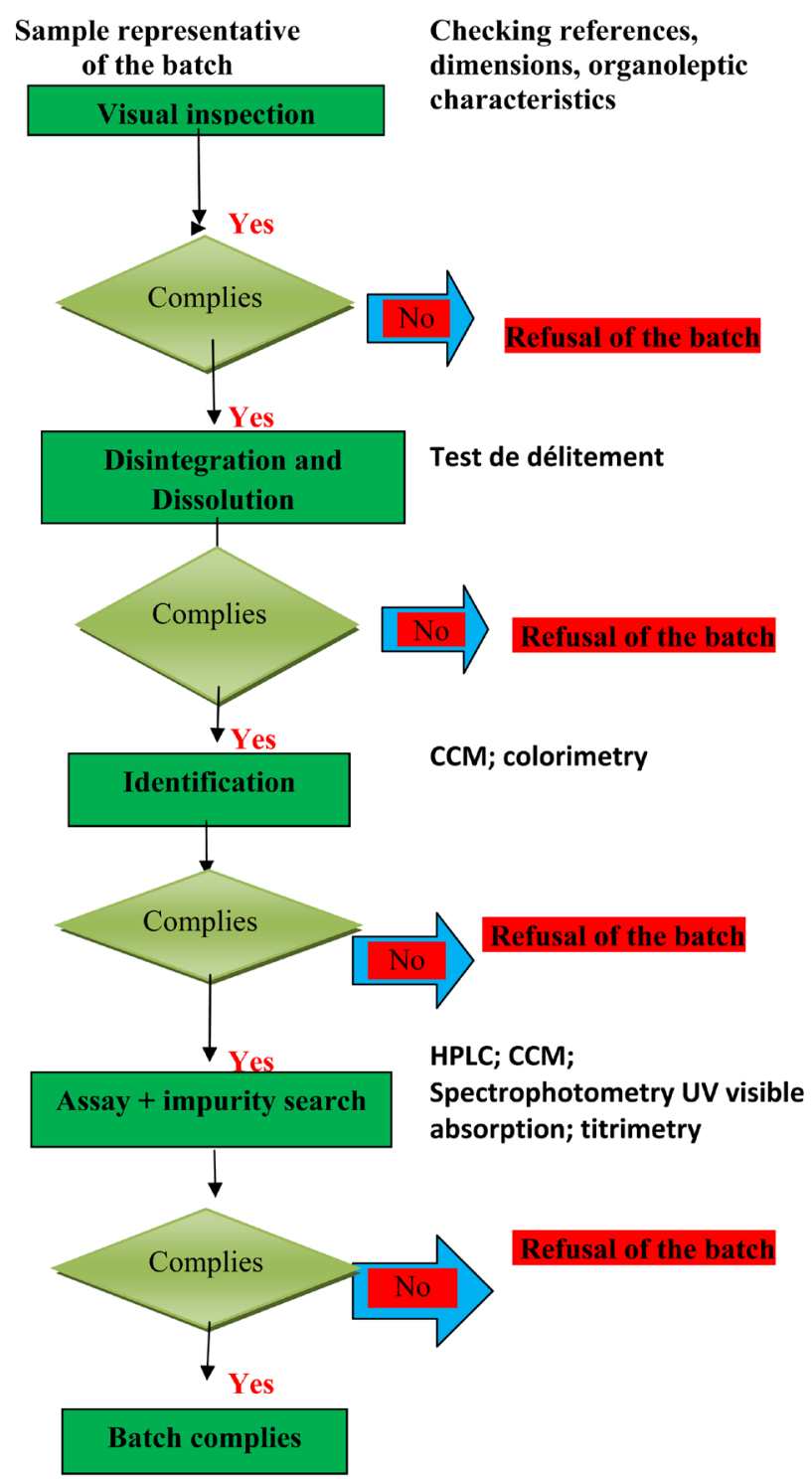

Figure 1. Analytical approach [14]. 


\section{Results}

The results of the study are summarized in Table 1.

This table shows that in 10 batches of artemether lumefantrine, 8 were found not compliant and that only half of lots of sulfadoxine pyriméthanmine complies.

Figure 2 highlights the percentages of non-compliance of the study. A percentage of non-compliance of $80 \%$ was noted in the case of artemether-lumefantrine combination.

\section{Discussion}

The interest of the study is justified first the high number of areas involved in illegal drug sales; and secondly by the development of resistance of $P$. falciparum to most antimalarial drugs. Also, the molecules are those targeted by the NMCP in Benin and the most sensitive people are children, pregnant women and the elderly. In Benin, the National Directorate of Public Health (DNSP) estimates that 85\% of Beninese are supplied by the illicit market.

Table 1. Non-compliance rate of samples for identification and dosage tests.

\begin{tabular}{|c|c|c|c|c|c|c|}
\hline Analysed Drugs & $\begin{array}{l}\text { Number of } \\
\text { analysed } \\
\text { batches }\end{array}$ & $\begin{array}{l}\text { non-compliant } \\
\text { identification }\end{array}$ & Under-dose & Overdose & $\begin{array}{l}\text { Without } \\
\text { active } \\
\text { principle }\end{array}$ & $\begin{array}{c}\text { Number of } \\
\text { non-compliant } \\
\text { samples }\end{array}$ \\
\hline Artemether-Lumefantrine & 10 & 3 & 5 & 0 & 3 & 8 \\
\hline Artesunate Amodiaquine & 10 & 0 & 2 & 2 & 0 & 4 \\
\hline Sulfadoxine-Pyrimethamin & 10 & 3 & 0 & 2 & 3 & 5 \\
\hline Quinine & 10 & 0 & 0 & 0 & 0 & 0 \\
\hline TOTAL & 40 & 6 & 7 & 4 & 6 & 17 \\
\hline Rate of non-compliance & - & $15 \%$ & $17.5 \%$ & $10 \%$ & $15 \%$ & $42.5 \%$ \\
\hline
\end{tabular}

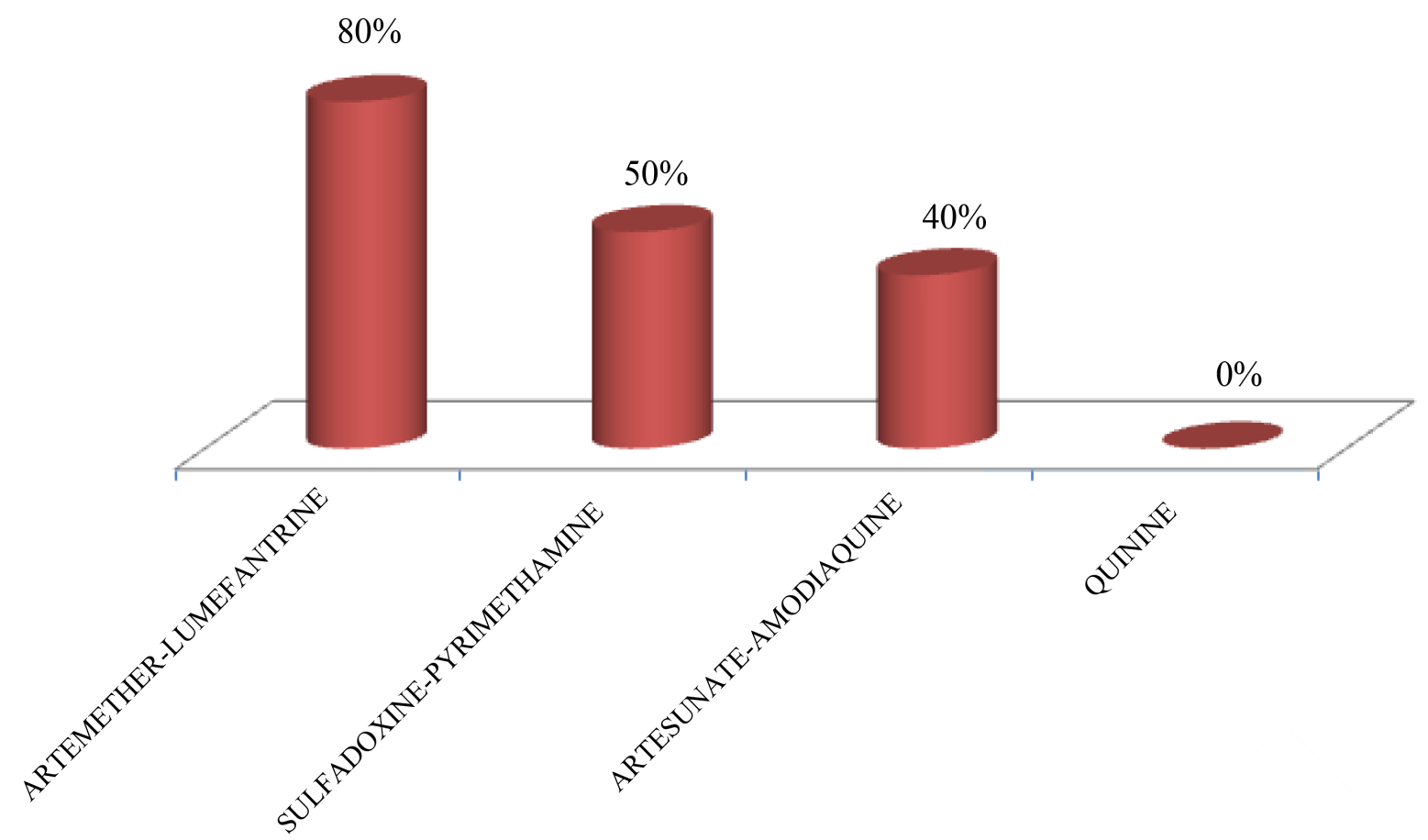

Figure 2. Rate of non-compliance samples. 
The macroscopic examination focused on the colour of the tablets, their shape and inscriptions on the surfaces of the tablet, as well as, the existence or not of the divisibility lines on the tablets. This control ended up without anomaly for all samples except for two batches of sulfadoxine-pyrimethamine. From sulfadoxine-pyrimethamine tablets of batch number EM 396, there were clearly tablets of various sizes. Also this batch of SP has two different manufacturing and expiry dates. This indicates a counterfeiting.

Furthermore, it was found a batch of sulfadoxine-pyrimethamine, batch number AG0080 marked "SAMPLE NOT FOR SALE” on the primary packaging. This may reflect an involvement of non-governmental organizations or medical representatives in the illicit sale of drugs.

All analysed samples were consistent with the mass uniformity test except for one batch of sulfadoxinepyrimethamine, batch number AG0080 containing 3 tablets which weights were above the highest limit of the average weight.

This mass unevenness reflects an individual weight difference of the sample units. From technical and regulatory view point, the sample is declared non-compliant. Such failure is often due to poor mixing and/or non-uniform filling of the compression dies during manufacturing due to the lack of knowledge either of these operations or the flow properties of the powders used to manufacture these drugs by the laboratories supplying this informal circuit [15] [16]. It could also be related to the significant erosion occurring during transport or storage in case the weight is lower than the minimum expected.

From the disintegration test, it was evaluated the approximate time that the drug will take to disintegrate once in the body.

With this test, one sample of sulfadoxine-pyrimethamine batch EM 304 was detected non-compliant among the 40 analysed samples, giving a rate of $5 \%$. This drug is disintegrated after almost an hour which is greater than the time interval prescribed by the Pharmacopoeia (normally equal or less than 15 minutes for naked and dried tablets). This is a danger to the consumer. The increase in the disintegration time can be observed in the case of a bad choice of formulation excipients, with insufficient amount of disintegration substance, a higher amount of binder, and an excessive compression of the tablet, inadequate coating system, alteration of the drug during storage [16]. Such situation is likely to lead not only to treatment failure, worsening of the clinical condition of the patient but also adverse effects on the digestive tract and kidneys.

At the identification test, 6 of the 40 analysed samples mainly, artemether-lumefantrine batches F1900, F0173, F1892 and sulfadoxine-pyrimethamine batches EM 396, EM 304 and AG0098 showed a total absence of active ingredient. The obtained compliance rate was $85 \%$. This rate is different from that obtained by Akole in Benin [9] where only 1 drug out of 53 presented a total absence of active ingredient with a compliance rate of $98.11 \%$ and the one obtained by Legris in Ivory Coast [17] where only one drug over 293 analysed contains no active ingredient having a compliance rate of $99.65 \%$.

The rate of absence of active ingredient is increasing. This decrease in the compliance rate is due to the proliferation of fraudulent laboratories. The absence of active ingredient in a drug is a manufacturing crime. These products devoid of the active substance or whose active ingredient was replaced by another ones are fake medicines and come often from illegal laboratories. They seriously affect the prognosis of patients resulting in treatment failure, sometimes poisoning and at worst death.

The dosage assay shows whether the active ingredient is present in sufficient quantity to produce the expected therapeutic effect. In the current study, 17 samples were detected non-compliant out of the 40 analysed giving a non-compliance rate of $42.5 \%$. This result correlates with that of Diallo conducted in Burkina Faso with a rate of 42\% [18] and that of Akole in Benin which came up with 24 samples over 53 with a non-compliance rate of $45 \%$ [19]. Nigeria and Thailand, have achieved $43 \%$ of non-compliance for the samples from Nigeria and $50 \%$ for those from Thailand. These rates confirm the non-compliance rates obtained in this study.

This rate is similar to that obtained by Basco [20], who studied the quality of antimalarials Cameroonians parallel market. It received $39 \%$ of non-compliant samples.

In detail, 17.5\% (7 of 40 samples) are under-dosed, 10\% (4 of 40 samples) are overdosed and 15\% (6 samples out of 40) do not contain any active ingredient. Considering only the results of the identification and dosage tests, the recorded non-compliance rate is $42.5 \%$.

This rate is lower than the one reported by Adognibo in Benin [21] with one conform sample out of three analysed giving a non-compliance rate of $66.66 \%$. This difference can be explained by the fact that in that study, the collected sample size is smaller than the current one and this could influence the obtained non-compliance. 
Also, the rate of non-compliance with sulfadoxine-pyrimethamine (12.5\%) is related to the one reported by the Institute of Research for Development of Cameroon, whereby a study on the quality of antimalarials collected from Cameroonian illicit market between 2001 and 2002 from street vendors revealed 12\% of sulfadoxinepyrimethamine samples as non-compliant [22].

Moreover, four boxes of artesunate-amodiaquine samples from batch number 5147 comply with pharmacopoeia standards. Despite their compliance, these drugs found in the illicit market were carrying the label of a certain local pharmacy. This could be explained by either the implication of pharmacy agents who divert drugs or insured clients who buy drugs at low cost and resell them to those in the informal markets.

Furthermore, none of the CTA batches found in the market is registered in Benin according to the National Programme against Malaria. These are probably escaped products from the formal distribution of one or more neighbouring countries or a very structured counterfeit circuit that reproduces them with the required standards.

All ten the batches of quinine found on the market comply with the various stages of analysis. However, 9 batches curiously had no manufacturing dates and come from Pharmaquick.

Although the compliance of those 10 batches of quinine reflects a respect of Good Manufacturing Practices, it obviously denotes a leak in the formal system. This situation can let patients doubt the legal system and could maintain self-medication and the orientation of populations towards the informal markets.

Under-dosing or overdosing of drugs may be due to a manufacturing defect, poor Manufacturing Practices: a mixture or a non-uniform filling of the compression dies during manufacturing [16] [23]. It may also be voluntary and deliberate act from the manufacturers themselves. The latter case is called pharmaceutical falsification.

Since the laboratories involved in the pharmaceutical counterfeiting operate in the informal sector, and that their products do not undergo any control by the drug regulatory authorities, they engage in acts of overdosing and under-dosing according to their objective.

The current study revealed four overdosed batches of drugs namely:

- Artesunate-amodiaquine batches 1370041 and LQ101019A

- Sulfadoxine-pyrimethamine batches 9CLT29 and AG0080.

In these cases, the objective of the manufacturers of these products could be to attract the consumer's attention on the effectiveness of their products. Patients taking these types of antimalarial will apparently feel more relieved in a short period of time and thus will tend to buy the same drugs.

Nevertheless, the negative aspects of such an act are the consequences on the health of the patient since overdosed drugs can be fatal for the patient if they have a low therapeutic index.

In addition, the dose ingested by the patient is greater than the recommended, and could thus have potentialtion of drug side effects, serious impact on the functioning status of vital organs, as well as, digestive disorders, peptic ulcer disease, hypoglycaemia etc.

Under-dosed medicines found in the six following batches:

- Artemether-lumefantrine batch F1882; K02386; F0359; P0400L

- Artesunate-amodiaquine batch 1370030, 6238.

Under-dosing together with the wrong directions for use given by the sellers, can favour the selection of resistant pathogens to authentic antimalarial drugs. They also cause a vulnerability of children and pregnant women to malaria. The health of the population is therefore threatened and many challenges await scientists in terms of resistance to antimalarial drugs. Concrete and actionable measures must be taken to eradicate this public danger.

Diseased people seek for medical consultations according to their prices, often difficult for low-income households, and the absence health facilities or the fact that they are far away from the households. The supply from the informal market offers lower costs than those of authorized pharmacies that provide high quality medicines.

Patients usually complain of high prices of products in pharmacies ignoring that street vendors products are tax free with no adequate follow-up due to the fraudulent origin of their commodities and they do not offer any health guarantee.

The illicit market offers no adequate storage conditions for medicines, which impairs their quality. However, it was found in the illicit market, some well stocked shelves with attractive arrangements and very friendly sellers willing to sell their fake products.

This remark is very scary and pharmacist should now avoid being absent from their workplace, develop a better quality service in order to avoid the risk of losing their role of public health actor. Also, according to the 
investigations conducted from the vendors they claim that their products are bought from NGOs, medical representatives, wholesalers' structures or in neighbouring countries. Such situation may alter the confidence in the health system. Therefore, health professionals, pharmaceutical companies and distributors that is engaged in those non-recommended practices and contrary to the pharmaceutical legislation must be combated and punished severely.

In view of these results, the research team decided to verify the quality of antimalarial drugs dispensed in the formal system basically in the local pharmacies. Therefore, previously reported data from the National Laboratory for Quality Control of medicines and medical consumables of the past three years were consulted. Fortunately, the observed average compliance rate is $98 \%$. This rate confirms the good quality of antimalarial sold in the formal system.

Overall, a drug with a positive disintegration test, identification and dosage test can be ineffective if the speed of release of its active ingredients does not comply with pharmacopoeia standards. It is therefore likely that the rate of non-compliance found in this study is lower than the reality. However, the tests that are performed in this study allowed appreciating the quality of the analysed samples.

Illegal trade and the free use of these non-compliant products encourage improper self-medication with incorrect dosing that ultimately leads to treatment failure. There is thus a need to be aware of the dangers associated with the presence of antimalarial drug in the illicit market mostly consumed by vulnerable people and that actions should be taken to eradicate this scourge.

At the place of pharmacists, periodically raise awareness of the dangers of the illicit market through regular television and radio programs for behavior change;

At the point of prescribing, educate patients disastrous consequences of street drugs;

At the place of NGOs and Medical Delegates, ensure sound management of donations of medicines and medical samples to prevent their escape into the informal circuit;

At the location of the population, procure drugs at the legal structures of drug distribution.

\section{Conclusions}

This study on the quality control of drug illicit market in Porto-Novo city revealed an overall non-compliance rate of $42.5 \%$ with $80 \%$ for artemether-lumefantrine, $50 \%$ for sulfadoxine-pyrimethamine, $40 \%$ for the artesunateamodiaquine and $0 \%$ for quinine. In the disintegration test, $2.5 \%$ of samples were found non-compliant; $17.5 \%$ are under-dosed; $10 \%$ are overdosed and $15 \%$ of samples are free of active ingredient. These results show that street vended drugs offer no guarantee of quality and are serious threat to the health of populations.

Also, the rate of non-compliance obtained denotes a flaw in the security of the distribution system. A study addressing the regulatory and analytical aspect involving other departments of the country with their various illicit drugs outlets would identify the extent and severity of this trade of fake medicines in Benin.

\section{References}

[1] WHO (1994) World Malaria Situation in 1994. Part I. Population at Risk. The Weekly Epidemiological Record, 72, 264-274.

[2] WHO (1998) Checklist: Malaria, No. 94. (In French)

[3] WHO (1997) Report on World Health: Life in the 21st Century-A Prospect for All. (In French)

[4] NMCP/MSP (2005) National Policy against Malaria and Implementation of the Strategic Framework. Cotonou, 50 p.

[5] Ministry of Health (2008) Statistical Yearbook of Health. Cotonou, Edition, 242 p. (In French)

[6] WHO (2010) Tests, Methods and Quality Standards for Substances, Excipients and Pharmaceutical Preparations. Geneva, (4): $362 \mathrm{p}$.

[7] Bagozzi, D. (2004) WHO Update on substandard and counterfeit medicines. World Health Organization, 1-8. (French).

[8] DPM/MSP (2007) Collection of Legislative and Regulatory Texts of Benin Pharmaceuticals Subsector. 3rd Edition, Cotonou, 239 p. (In French)

[9] Akole, C. (2010) The Fight against Counterfeit Drugs: Quality Control of Drugs Pilot Study of Benin Illicit Market. Pharmacy Doctoral Thesis, Faculty Pharmacy/FSS/C.

[10] International Pharmacopoeia (1994). 3rd Edition.

[11] American Pharmacopoeia. (2000). 24th Edition NF19. 
[12] European Pharmacopoeia (2005). 5th Edition, Volume 1.

[13] American Pharmacopoeia (2011) 34th NF29 otonou , No. 058, 85. (In French)

[14] Baba-Moussa, F. (2005) Personal Communication: Sub Regional Consultation of National Quality Control Laboratories. (In French)

[15] Pradeau, D. (1992) Analysis Practice of Medicine. International Medical Publishing, 1066 p. (In French)

[16] Fonteneau, J.M., Orecchioni, A. and Pain, J. (1999) Galenics, Pharmaceutical Operations, Concept of Drug-Dosage, 209 p. (In French)

[17] Legris, C. (2005) The Detection of Counterfeit Drugs through Investigation of Their Authenticity. Pilot Study on the Illicit Drug Market in Ivory Coast. PhD in Pharmacy, Nancy I., 98 p. (In French)

[18] Diallo, S., Maike, T. and Coulibaly, B. (2008) Substandard Anti-Malarial Drugs in Burkina-Faso. Malaria Journal, 7 , 95. (In French) http://dx.doi.org/10.1186/1475-2875-7-95

[19] Shakoor, O., Taylor, R.B. and Behrens, R.H. (1997) Assessment of the Incidence of Substandard Drugs in Developing Countries. Tropical Medicine \& International Health, 2, 839-845. http://dx.doi.org/10.1046/j.1365-3156.1997.d01-403.x

[20] Basco, L. (2004) Molecular Epidemiology of Malaria in Cameroun. Quality of Antimalaria Drugs Used for SelfMedication. American Journal of Tropical Medicine and Hygiene, 70, 245-248.

[21] Adognibo, I. (2008) Quality Control of Artemether-Lumefantrine in Health Facilities in Benin. PhD in Pharmacy, Pharmacy UFR/FSS/Cotonou, No. 031, 92. (In French)

[22] Koffi, L., Konan-Macy, M. and Yebouet, B. (1999) The Acute Poisoning in Pediatrics at the University Hospital of Yopougon, Ivory Coast. Bulletin of the Exotic Pathology Society, 2, 114-117. (In French)

[23] Blazy, A. (2002) Unused Medicines and Humanitarian Aid: Evolution and Current Situation. Pharmacy Doctoral Thesis, Lyon. (In French) 